\title{
Towards Better Team dynamics for International Projects in Belt and Road Countries
}

\author{
Ying Zhang \\ 1. International Business School \\ Yunnan University of Finance \& Economics \\ 2. School of Management and Marketing \\ Charles Sturt University, Wagga Wagga, Australia \\ angie@ynufe.edu.cn
}

Xialing Wei

International Business School,

\begin{abstract}
Yunnan University of Finance and Economics, 286145889@qq.com
\end{abstract}

\author{
Tiffany Tonge* \\ International Business School \\ Yunnan University of Finance \& Economics \\ Kunming, China \\ tiffany.tonge@me.com
}

\begin{abstract}
This paper offers an aggregate view of emotional intelligence (EQ), team dynamics and innovative climate within multicultural teams on international projects in belt and road countries. The main objective is to establish whether there is a mediated relationship between emotional intelligence and team dynamics, through climate for innovation, in order to give recommendations for multicultural project management in belt and road countries. The paper elaborates on the relationships that were found to be vital and significant. Result shows no immediate and obvious mediation effect of climate for innovation on the relationship between emotional intelligence and team dynamics. Conclusion demonstrates that although emotional intelligence has some attributes that can contribute to creating an atmosphere where team dynamics can be more prevalent, it is also not an antecedent to the commitment on multicultural teams.
\end{abstract}

Keywords-emotional intelligence; innovation; team dynamics

\section{INTRODUCTION}

There is a critical need for the project team leader to create a climate for innovation when managing international projects in belt and road countries so as to succeed and thrive in the multicultural environment. There are factors that suggest the critical need for the project teams to create a climate for innovation when managing international projects, in order to succeed and thrive in the multicultural environment.

With regard to the labor side of the projects, the emotional intelligence competency can be instrumental in fostering growth in one of the very foundational keys of project and organizational success, which is team dynamics [1]. The success of these types of development projects may rest entirely on the team dynamics and the cooperation of the team, between key persons on national and international project teams who manage the different processes and moving parts of these projects. Because of the fragile financial condition for most of these developing countries, the onus in on the government stakeholders, project committees and other major stakeholders, to invest their interest in methods that will maximize the productivity of the use of these scarce resources,

*Corresponding author in order to derive high value and economic gain from the project, and to be successful with little to no wastage of finance, time and labor resources.

There is a general observation that the globalization of the economy all over the world has made the world to become a small global village where the international organisations rely on multicultural teams to perform creative team work, in order to meet the modern challenges of a complex business environment. Project managers that are highly skilled and with high emotional intelligence skills are needed to work with such culturally diverse team members as this trend continues. This paper assesses the theoretical framework of how EQ and team dynamics are related. The aim of our study is to investigate whether there is a mediating effect of climate for innovation on this relationship using the facts in literature and also presents the findings of a quantitative research that was based on primary data collection and analysis.

\section{LITERATURE REVIEW AND HYPOTHESES}

\section{A. Emotional Intelligence}

Study on emotional intelligence is led by two major schools of theorists, Salovey \& Mayer and the team of Daniel Goleman \& Richard Boyatsis [2]. Salovey and Mayer proposed their theory of emotional intelligence in 1990. These theorists relied on a tool from the Mayer-Salovey-Caruso Emotional Intelligence test (MSCEIT) to test their specific hypotheses and conclusions. Goleman and Boyatsis proposed inductively on the basis of the theory of emotional intelligence, which was tested with the emotional competence of the inventory (ECI). Both theories are similar in that they each suggest that awareness and regulation of emotions at the individual and social level are critical for team performance.

Goleman and Boyatsis [3][4] pioneered the development of four separate clusters. In addition, Salovey and Mayer suggest that companies used to apply emotions to solve problems are an additional component of an individual of emotional intelligence [2][4]. Thus, the competence encompasses 
personal characteristics (traits, understood school attributes and emotional intelligence) of skills and knowledge (including mental ability and the ability of finding fast solution for the problems, as well as control skills). Therefore, such competence covers characteristics that are considered as personal such as knowledge and skills that is comprised of ability to think and reason out issues and the ability to solve problems. Consequently, emotional competence is the most significant, but two other important ones, as suggested by Barnard and Confucius [3]. Research has also shown that it serves as an important tool, which greatly benefits the multicultural project team because it builds greater team cohesion than teams with lower average emotional intelligence [3][4].

\section{B. Emotional Intelligence and Team Dynamics}

The successful management of diversity can benefit an organization by building emotional competence and can be used in turn to connect individuals of different backgrounds in order to build cooperative and successful teams within the organization[2][4]. This in turn can enhance team dynamics, because team members with higher levels of EQ would have in mind mutual cooperation, and can have a tremendously positive impact on multicultural project teams. Chang et al. [5] stressed the importance of team dynamics and emphasized that the greater the number of individuals in the team, the greater the probability of conflicts and misunderstanding.

This gives the team a sense of acceptance and belonging, where they feel that they can contribute to the success of reaching the goals by investing their ideas and energies into the organization, thus fostering an attachment to the organization for the mere reason of seeing their solutions come to fruition. Prior studies [2][3] expressed the need for further multi-level research in this area that examines the leader EQ competencies and the development of organizational EQ competencies. This suggests the area is still young and more developmental studies need to take place so that its assumptions and the benefits can be concretized.

\section{The Relationship Between Emotional Intelligence and Climate for Innovation}

Creating a climate for innovation after the conception of an idea, rests squarely on the ability of the team and the team leader's ability to recognize a need for change and the need to search for relevant problem solvers in the team, while lending support for the group and encouraging them to come above the status quo[4]. One of the major factors that can contribute to this understanding and openness is being able to successfully communicate to teams in a way that will allow them to see that ideas are welcome [2]. In a multicultural team, being emotionally competent can fill the gap, and pave the way of the implementation of ideas on the team.

Innovation in multicultural teams is a subject that needs attention because the world has increasingly become a global village. Having the emotional intelligence competency is one that brings to the table respect of diversity and appreciation for new ideas that come to the fore. This competency would allow for more flexibility and tolerance for differences, because of different backgrounds, values and belief systems, and brings to the fore, the positives of new knowledge because of experience, and being able to stimulate and atmosphere of trust in order for person to work cohesively and to come forward with new solutions [3][4].

\section{Climate for Innovation and its Mediating Relationship with Team dynamics}

The innovative support in organizations (and by extension it's leaders, because they are the decision-makers), are positive predictors of affective and continuance team dynamics [5]. Organizations that support innovative endeavors increase a shared sense of success in meeting the goals of the organization and add to the cohesiveness in teams. Team cohesiveness is a significant factor to increased productivity and any increase in productivity, leads to higher profitability, saved time more efficiently used resources and keeps the project on the road to delivering what was agrees to, within budget and in the correct quality. Other studies have shown that even beyond influence, the climate for innovation is significantly related to team dynamics [4][5].

The climate for innovation within organizations also builds autonomy, empowerment and confidence of the team members; this makes them more likely to be motivated to work well and to influence them to identify with the organization and what the goals are[3][5]. It is however argued that team dynamics is proportional to the extent to which team members feel that their contribution is valuable or utilized the organization [6]. This can foster team dynamics as a state in which an employee identifies himself within a particular innovative climate, and wishes to maintain membership with that organization. This demonstrates the importance of team leaders creating a culture within their group, which fosters this type of commitment. When there is support for innovation, where employees are encouraged to make individual contributions, and are given the autonomy to work on tasks, then they have a sense of being able to contribute to the organization's success and therein become more affectively committed to the organization [4] [6].

\section{E. Emotional intelligence, Innovation and Team dynamics}

There are several benefits that team members and leaders when they have the competency of emotional intelligence; they are able to facilitate team performance by their ability to facilitate communication and cooperation, which being about to foster and environment of trust, openness and support for different perspectives according to working experience and different backgrounds[6]. This would create an open-minded atmosphere where the sharing of knowledge and ideas in encouraged and therefore creates a productive space for growth and cohesion.

The climate for innovation could be therefore formed, because these are the very factors that influence and promote the environment of creativity. Although the emersions of ideas is not constrained to a multicultural environment, as innovation can occur in a mono cultural context, there are many novel ideas that can contribute to successfully sharing knowledge and working to implement these so that undertakings can share more success [3] [6]. With this support for creativity, in mind, 
team leaders and team mangers should ensure that there are sufficient resources made possible in order to assist the implementation of ideas. This may promote to the employees having incentive to be more productively creative and can positively influence their identity with the organization.

When employees begin to identify with the organization, this gives rise to affective and continuance dynamics; although the climate for innovation is not the only factor that influences team dynamics, but also contributes to the way the employees' feel when they are able to contribute to, and implement ideas and within their teams, it gives them a sense of ownership, of their particular implementation and therefore their desire to remain within the organization [5][6]. With these factors in place, this may give rise to the team members' sense of continuance commitment where they want to see their implementation through not only for the short term but also for the long term.

\section{F. Theoretical Framework}

Upon investigating the literature it was found that the successful management of diversity can benefit an organization by building cross-cultural competence and can be used in turn to connect individuals of different backgrounds in order to build cooperative and successful teams within the organization [3]. When there is support for innovation, where employees are encouraged to make individual contributions, and are given the autonomy to work on tasks, then they have a sense of being able to contribute to the organization's success and therein become more affectively committed to the organization [5].

This gives weight to the current study where these relationships will be hypothesized to make a framework that will test for a mediating relationship of climate for innovation on EQ and dynamics, and to establish whether it is an antecedent to achieving team dynamics in multicultural project teams. Based on foregoing analysis, we speculate that greater the emotional intelligence, the climate for innovation will be stronger in teams, which in turn will increase team dynamics. Therefore,

Hypothesis: The climate for innovation will mediate the relationship between emotional intelligence and the team dynamics of multicultural team.

\section{ReSEARCH Methodology}

The study adopted a quantitative approach, through a survey research design that was correlational in its approach and cross sectional in its scope. This study used the nonprobability-sampling frame within the Project implementation team population. The sample for the study consisted of 108 team leaders and technical staff of multicultural teams from both public and private sector organizations.

The survey tools used in this research are those that have been used in past studies pertaining to the variables that are being examined in this study; all were placed within one survey for participants to answer. The data collected in this survey, was derived from a survey instrument, distributed electronically, using these instruments of quantitative analysis for measuring all dimensions of emotional intelligence, team dynamics, and the climate for innovation (CI) scale. Respondents completed the questionnaire on a 5-point scale (1 strongly disagree; 5 strongly agree). A pilot study was carried out before the instruments were re-worked to suit the situation of the persons to participate in the survey.

A simple mediation analysis through the causal path method was used for this analysis; included in this method, was the bivariate analysis using the Pearson's coefficient correlation analysis and a final regression was used to complete the analysis.

\section{DATA ANALYSIS AND RESUlTS}

\section{A. Descriptive Statistics}

The study comprised of 110 participants from the project management population within belt and road countries of China, the Philippines and Malaysia. The sample consisted of 68 Females, which represented majority of the sample and had a corresponding percentage of 61.8. In contrast, the male category represented the minority of the sample and accounted of 42 participants, which had an associated percentage of 38.2. In addition to gender, the age demographic was subdivided into 5 groups, with the age group 30-39 years representing majority of the sample ( $\mathrm{n}=58,52.7 \%)$, followed by the age group Under 30 years $(n=22,20 \%)$, then the age group $40-49$ years $(\mathrm{n}=18,16.4 \%)$.

The age group 50-59 years $(\mathrm{n}=9,8.2 \%)$ was the smallest category only to the Over 60 age group, which represented the minority of the sample $(n=3,2.7 \%)$. The highest education category received an overwhelming number for participants who had acquired a Masters' degree $(n=41,37.3 \%)$. This was followed by those who had a Post Graduate degree $(n=34$, $30.9 \%$ ) and subsequently participants who had an undergraduate degree $(\mathrm{n}=29,26.4 \%)$.

\section{B. Pearson's Correlation Coefficient between Emotional Intelligence and Innovative Climate}

A Pearson's correlation coefficient was conducted to evaluate the relationship between emotional intelligence and innovative climate. The results show that there was very weak negative relationship between emotional intelligence and innovative climate indicated by $(n=110 \mathrm{r}=-.016, \mathrm{p}>.05)$, this shows that this is not statistically significant. The relationship between emotional intelligence and team dynamics the Pearson's product-moment coefficient was computed to evaluate the relationship between emotional intelligence and team dynamics, the results show that there was an extremely weak negative relationship that was not significant $(n=110, r=-$ $.006, \mathrm{p}>.05$ ). Finally, a Pearson's product moment correlation coefficient was conducted to evaluate the relationship between innovative climate and team dynamics. The results indicated that there was a moderate to strong relationship that was positive and also statistically significant $(n=110, r=.454$, $\mathrm{p}<.05)$ 


\begin{tabular}{|l|c|c|c|}
\hline \multicolumn{1}{|c|}{ Variables } & 1 & 2 & 3 \\
\hline 1.Emotional intelligence & 1 & -.016 & -.006 \\
\hline 2.Innovative Climate & -.016 & 1 & $.454^{* *}$ \\
\hline 3.Team dynamics & -.006 & $.454^{* *}$ & 1 \\
\hline
\end{tabular}

\section{TABLE I. PEARSON'S CORRELATION COEFFICIENT RESUlT}

\section{Regression Analysis}

To assess the causal path mediation, a regression analysis was used. Four regression analysis were computed, the first regression analysis assessed path (a), which showed a standardized data coefficient of -.016 from emotional intelligence to climate for innovation. The second regression path showed a (b) path, which highlighted a .454 standardized data coefficient, indicting a significant positive relationship between climate for innovation and team dynamics. Thirdly (a1) showed that there was a weak positive relationship that was not statistically significant this was discovered after emotional intelligence was tested through the first (a) path to climate for innovation toward team dynamics (b), the effect was reduced and also the direction of the relationship changed (.001). However according to Barron and Kennedy's [7] criteria for mediation analysis, it requires that all paths have a statistically significant findings, this is not the case for this causal model path (a) and path (a1), do not suggest that they are significant and thereby suggests that not mediation can be derived from climate for innovation with respect to the following results.

TABLE II. REGRESSION PATH COEFFICIENTS

\begin{tabular}{|l|r|r|r|r|r|}
\hline Coefficient & $\boldsymbol{U B}$ & $S D$ & $\boldsymbol{B}$ & $\boldsymbol{t}$ & sig \\
\hline (Constant) & 24.373 & 6.485 & & 3.758 & 0 \\
\hline $\begin{array}{l}\text { Innovative } \\
\text { Climate }\end{array}$ & 0.486 & 0.092 & 0.454 & 5.266 & 0 \\
\hline $\begin{array}{l}\text { Emotional } \\
\text { intelligence }\end{array}$ & 0.001 & 0.072 & 0.001 & 0.015 & 0.988 \\
\hline a. Dependent Variable: Team dynamics & & & & \\
\hline
\end{tabular}

Note: $\mathrm{UB}=$ Unstandardized coefficient Bata; $\mathrm{SD}=\mathrm{Standard}$ error; $\mathrm{B}=$ standardized coefficient Bata

\section{RESULTS AND DISCUSSION}

\section{A. Emotional intelligence and Climate for Innovation}

The result of this study has shown that there was weak negative relationship between these two variables $(n=110, r=-$ $.016, \mathrm{p}>.05)$. According to literature, it was discovered that emotional intelligence on teams that are multicultural, gives way to higher climate for innovation because it can contribute to a higher level of understanding, communication and atmosphere of trust, within teams that are made up of person's from different backgrounds. Prior studies demonstrate no evidence that emotional intelligence is an antecedent to the climate for innovation. This means, that whilst it is good to have emotional intelligence within multicultural teams it is not necessary for the increase of the climate for innovation.

\section{B. Climate for Innovation and Team Dynamics}

The test for a relationship between climate for innovation and team dynamics prove to be different than the previous test $(n=110, r=.454, p<.05)$. This result suggests that the higher the climate for innovation, the higher the team dynamics in these teams The Pearson's correlation coefficient test was done between climate of innovation and team dynamics and it was found to have a strong positive correlation. This result reflects what prior studies have shown with respect to these two variables.

The team dynamics that happened as a result would have caused persons to identify with the organizations to be more highly motivated to participate in decision-making, and feel their skills to be adequately utilized by the organization, and therefore empowers them to be more productive. These assists in project implementation because employees perceive themselves to be valued by the organization and therefore their teams would then use their thoughts, suggestions and solutions. This indicates that when teams have a support for innovation it will then foster a sense of shared values goals and success amongst the team members and therefore gain higher levels of team dynamics which would lead to more efficiently use the resources within projects.

\section{Emotional Intelligence and Team Dynamics}

Within the context of this study, it has been the result that emotional intelligence and team dynamics has an insignificant relationship according to the Pearson's coefficient correlation analysis that was done. This test yielded two results, the first result that was yielded was a weak negative insignificant result before regression $(\mathrm{N}=110, \mathrm{r}=-.006, \mathrm{p}>.05)$ and after regression, the result changed to (a1) $\mathrm{r}=.001$, which signifies a weak positive relationship with team dynamics. Therefore, emotional intelligence does not show any variation with team dynamics, which contradicts to our speculation. Further to this, other studies have found results that conform to this same research pertaining to these two same variables, showing that relationships involving team dynamics tend to be less influenced by emotional intelligence. When considering this result and for the study within literature in the context of multicultural teams within belt and road countries, it shows that emotional intelligence is not an antecedent for team dynamics there are many other factors that can affect team dynamics.

In light of this it is important to note that emotional intelligence helps to manage conflict within the organization. On multicultural teams it helps to build the factors of success in communicating the team goals values and tasks. It creates the environment where when it is used effectively in teams, then an environment of cohesion is formed, and therefore employees are motivated to continue to work together to work to that common goal. These are factors that contribute to the antecedents of team dynamics but emotional intelligence itself is not necessary for these conditions to happen. 


\section{CONCLUSION AND RECOMMENDATION}

This paper examines how team leader can maximize its economic value of the team by obtaining team dynamics through creating an atmosphere for innovation in multicultural project teams especially for those in developing countries.

Emotional intelligence adds to the organizational climate but it is not an antecedent to successful interactions, creativity and innovation within multicultural teams. It however raises the tolerance of differences, open communication, sharing of knowledge and ideas etc.

The factors that cause these attributes that contribute to climate for innovation seem to exist outside of EQ it is an important complementary competency as it relates to being able to interact successfully with persons of other cultures. However it is noted that emotional intelligence adds to the organizational climate but it is not an antecedent to successful interactions, creativity and innovation within multicultural teams. It was found in prior studies that training for cultural competence may not be as effective as having an experience of immersion within another culture; this takes time and resources and may not be practical for locals who work with multicultural teams.

With respect to the results of this study it seems that creating an atmosphere within the organization that fosters a safety and trust would be the most important aspect in creating a climate for innovation and is thereby strengthening team dynamics. Finally, team leaders and team members of multicultural teams are a part of a multifaceted phenomenon that has many different factors that produces organizational citizenship and thereby increasing productivity and reducing waste of resources. It is imperative that research studies look at other as variables that would team up with emotional intelligence to mediate some moderate the effect team dynamics within multicultural projects.

Future research should consider a longitudinal study, whereby patterns and sequences can be found throughout time and across sections, thereby introducing further more complex analysis like structural equation modeling, and being able to establish cause and effect relationships. It can use a larger sample size over the whole Silk Road region; this would increase statistical power of the research. Another area that would be useful for future research is that these variables should be examined within the expatriate context, instead of in the context of the host country and should investigate the effects of emotional intelligence on teams where there are culture specific tasks.

\section{ACKNOWLEDGEMENT}

Funding for this research was provided by National Natural Science Foundation of China under grants 71762033. The international collaborative research program with Charles Sturt University in Australia is gratefully acknowledged. The assistance of Australian scholars Dr. Sophia X. Duan and Professor Mark Frost are also acknowledged.

\section{REFERENCES}

[1] G. Eason, B. Noble, and I.N. Sneddon, "On certain integrals of Lipschitz-Hankel type involving products of Bessel functions," Phil. Trans. Roy. Soc. London, vol. A247, pp. 529-551, April 1955.

[2] G. Matthews, M. Zeidner, et al. Emotional intelligence, science and myth, Cambridge, MA: MIT Press, 2002.

[3] Goleman, Boyatzis, et al. Primal Leadership: Learning to Lead with Emotional Intelligence, Harvard Business School Press, Boston, MA, 2002 .

[4] D. Goleman. Emotional Intelligence, Bantam Books, New York, NY,1995.

[5] J.W. Chang, T. Sy, J.N. Choi. Team emotional intelligence and performance: Interactive dynamics between leaders and members.[J]. Small Group Research, 2012, 43(1):75-104.

[6] W. Kang, A.M. Low, W. Yong. Effects of emotional intelligence on team dynamics with team leadership and communication as mediators $[\mathrm{J}]$ 2006.

[7] R.M. Baron,. \& D.A. Kenny, The moderator-mediator variable distinction in social psychological research: Conceptual, strategic, and statistical considerations. Journal of Personality and Social Psychology, 51,pp.1173-82,1986 